\title{
论 文
}

\section{光阑对暗中空涡旋光束轨道角动量的影响}

\author{
吕宏，柯熙政*
}

西安理工大学自动化与信息工程学院, 西安 710048

*联系人, E-mail: xzke@263.net

收稿日期: 2010-11-28; 接受日期: 2011-05-12; 网络出版日期: 2011-06-13

国家自然科学基金(批准号: 60977054)、国防重点实验室基金(编号: 9140C3601010901)、国家高技术发展计划(编号: 2008AAJ159)、陕 西省教育厅产业化项目、西安市科技攻关项目、陕西省知识产权局基金项目和陕西省自然科学基金(编号: 2007F12)资助项目

摘要 基于推广的复高斯函数展开法, 分析了具有轨道角动量的拉盖尔-高斯(Laguerre-Gaussian, LG)光束 通过含光阑光学系统传输的解析公式, 理论推导了空心 LG 光束在柱坐标系下光子轨道角动量密度函数及其 态矢. 对 1 阶 LG 涡旋光束通过光阑后的光场分布情况进行了数值分析，在两种典型含光阑系统物理模型下分 析了遮拦比对不同波长 LG 光束传输特性的影响, 研究了 LG 光束通过光学系统后的相位分布及轨道角动量密 度变化. 结果表明, 光阑系统遮拦比对 LG 涡旋光束通过光阑后的传输特性的影响因光束波长不同而异, 遮拦 比对光束轨道角动量密度影响较大, 对光束束径及光轨道角动量影响不明显. 研究结果可为在空间光通信中 利用涡旋光束光子轨道角动量编码信息提供理论依据.

关键词光束传输, 光学涡旋, 轨道角动量, 光阑

PACS: $\quad$ 42.60.Jf, 42.50.Tx, 42.79.Ag

当激光光束含有角向相关的位相分布(扭转位相 或螺旋位相)时就具有与角向位相分布有关的轨道角 动量, 携带轨道角动量的光束被称为 “光学浴 旋”(Optical Vortices, OV $)^{[1 \sim 4]}$. LG 光束就是一类典型 的浴旋光束, 具有固有的光轨道角动量 ${ }^{[5]}$, 在传播方 向上每光子的轨道角动量值为弧向量子数 $l$ 与约化 普朗克常数 $\hbar$ 的积的形式, 表示为 $l \hbar$. 具有轨道角动 量的光束具有更多的自由度, 利用光轨道角动量编 码相对于传统的二进制编码能够有效地提高数据传 送密度和速率 ${ }^{[6]}$, 而且可能的光束轨道角动量数会受 到如光学孔径等实际条件的限制. 每光子轨道角动 量可以用来定义无限维的希尔伯特空间 ${ }^{[7]}$, 创建高维 纠缠态以实现高效的量子信息处理.
无线激光通信的光调制、准直过程中, 光束通常 需要通过含有透镜以及光阑等光学元件的光学系统, 而单透镜本身的边框也是孔径光阑, 光束在传输过 程中总会受到光阑的限制. 在最近发表的文章中 ${ }^{[8]}$, 我们提出利用具有轨道角动量无衍射光的空间传输 特点, 将携带信息的 LG 光转换成具有相同拓扑荷的 高阶 Bessel无衍射光实现数据编码解码方法. 为了将 涡旋光束所具有的轨道角动量用于空间光通信, 研 究浴旋光束通过含光阑透镜光学系统后的轨道角动 量特性具有重要的意义. 2002 年, 王喜庆等人 ${ }^{[9]}$ 推导 了无光阑限制情况下聚焦 $\mathrm{LG}$ 光束的相对光强增量和 焦移, 对有光阑限制的 LG 光束的聚焦特性进行了数 值计算. 彭润伍等人 ${ }^{[10]}$ 推导了 $L G$ 光束通过光阑透镜

引用格式: 吕宏, 柯熙政. 光阑对暗中空浴旋光束轨道角动量的影响. 中国科学: 物理学 力学 天文学, 2011, 41: 932-941 Lü H, Ke X Z. Effects of aperture on the orbital angular momentum of hollow vortex beam (in Chinese). Sci Sin Phys Mech Astron, 2011, 41: 932-941, doi: 10.1360/132010-1149 
分离系统的光强分布, 利用数值计算分析说明了光 束的聚焦特性, 得出光阑透镜分离程度的大小影响 相对焦移的大小和光强的分布, 并引起焦移可能相 反或消失. 2006 年, 葛卫国等人 ${ }^{[11}$ 基于厄米-高斯模 与拉盖尔-高斯模的变换关系和递推算法, 推导出 LG 光束通过含矩形光阑近轴 $\mathrm{ABCD}$ 光学系统传输的递 推公式, 对 LG 光束通过光阑透镜分离系统的变换作 了数值计算和分析. 2007 年王备战等人 ${ }^{[12]}$ 推导了空 心高斯光束通过受圆孔硬边光阑限制的傍轴 $\mathrm{ABCD}$ 光学系统后的传输公式, 研究了光阑、透镜以及光阑 透镜系统对空心高斯光束传输特性的影响. 文献[13] 通过计算光束的二阶强度矩, 给出了轨道角动量和 二阶矩之间的关系. 目前研究多是针对 LG 光束的聚 焦特性展开的, 并未考虑光束的轨道角动量及位相 因子, 对于 $L G$ 光束通过典型光学系统后光子轨道角 动量密度变化情况的研究也未见报道.

本文基于推广的复高斯函数展开法, 分析了 LG 暗中空光束通过含圆孔硬边光阑限制光学系统的传 输特性, 推导了柱坐标系下单光子轨道角动量密度 函数及在某一范围空间的分布概率. 通过对 1 阶 LG 空心光束通过不同光阑系统后的场强分布进行详细 的数值计算, 分析了光阑孔径遮拦比对不同波长 $\mathrm{LG}$ 光束近场传输场强分布的影响, 同时讨论了遮拦比 对通过光学系统后单光子轨道角动量密度的影响. 研究结果对利用浴旋光束的轨道角动量携带信息实 现空间光通信提供一定理论依据.

\section{$1 \mathbf{L G}$ 浴旋光束通过含光阑光学系统的传输}

Collins 采用近轴矩阵光学方法 ${ }^{[14]}$, 对轴对称光 学系统用轴对称光学系统的变换矩阵 (ABCD 矩阵) 表示. 设光线顺次通过的光学系统的 $\mathrm{ABCD}$ 矩阵分 别为

$$
M_{1}=\left(\begin{array}{ll}
A_{1} & B_{1} \\
C_{1} & D_{1}
\end{array}\right), M_{2}=\left(\begin{array}{cc}
A_{2} & B_{2} \\
C_{2} & D_{2}
\end{array}\right),
$$

其中 $A, B, C, D$ 为傍轴光学系统的变换矩阵元. 柱坐 标下高斯光束通过由 $\mathrm{ABCD}$ 矩阵 $M_{1}$ 与 $M_{2}$ 表示的 复杂光学系统 $M$ 后, 在传输距离 $z$ 处的场分布可表 示为

$$
E(r, \theta, z)=\frac{\mathrm{i}}{\lambda B} \exp (-\mathrm{i} k z) \int_{0}^{2 \pi} \int_{0}^{\infty} E_{0}\left(r^{\prime}, 0\right)
$$

$$
\times \exp \left\{-\frac{\mathrm{i} k}{2 B}\left[A r^{\prime}-2 r^{\prime} r \cos \left(\theta-\theta^{\prime}\right)+D r^{2}\right]\right\} r^{\prime} \mathrm{d} r^{\prime} \mathrm{d} \theta^{\prime},
$$

其中 $k$ 为波数, $k=2 \pi / \lambda, \lambda$ 为波长, $E_{0}\left(r^{\prime}, 0\right)$ 为入射 参考面处的场分布, $r$ 与 $r^{\prime}$ 分别为接收面与入射面内 径向距离, $\theta$ 为位相角, 利用乘法规则有

$$
M=\left(\begin{array}{ll}
A & B \\
C & D
\end{array}\right)=\left(\begin{array}{ll}
A_{2} & B_{2} \\
C_{2} & D_{2}
\end{array}\right)\left(\begin{array}{ll}
A_{1} & B_{1} \\
C_{1} & D_{1}
\end{array}\right) .
$$

LG 光束具有固有的光轨道角动量 $l \hbar$, 其中整数 $l$ 又称作光学涡旋的拓扑荷 (Topological Charge), 当 $l \geqslant 1$ 时光束中心是暗的, 这是由于相位奇点导致强 度为零, 并且 $l$ 值越大 $\mathrm{LG}$ 光束直径越大. 在柱坐标 $(r, \theta, z)$ 情况下, $\mathrm{LG}$ 光束在 $z=0$ 处的场分布为 ${ }^{[15]}$

$$
\begin{aligned}
E\left(r_{0}, \theta_{0}, 0\right)= & \left(\frac{\sqrt{2} r_{0}}{w_{0}}\right)^{l} \exp \left(\frac{r_{0}{ }^{2}}{w_{0}{ }^{2}}\right) \\
& \times L_{p}^{l}\left(\frac{2 r_{0}{ }^{2}}{w_{0}{ }^{2}}\right) \exp \left(-\mathrm{i} l \theta_{0}\right),
\end{aligned}
$$

其中 $r_{0}$ 和 $\theta_{0}$ 分别为径向和角向坐标, $w_{0}$ 为束腰宽度, $L_{P}^{l}$ 为缔合拉盖尔多项式, $l, p$ 是标定 $L G$ 模式的特征 量子数, 是整数变量, $\exp (\mathrm{i} l \theta)$ 是位相因子, 表明是 一种具有螺旋结构的光波. 当弧向量子数 $l=1$, 径向 量子数 $p=0$ 时, $L G$ 光束是单环暗中空光束, 表示为 $L G_{0}^{1}$.

对 $L G$ 光束通过含硬边光阑近轴 $A B C D$ 光学系 统的场分布近似解析公式进行推导, 将光阑窗口函 数展开为有限数目的复高斯函数的叠加, 有窗口 函数:

$$
H\left(r_{0}\right)=\sum_{J=1}^{N} A_{j} \exp \left(-\frac{B_{j} r_{0}{ }^{2}}{a^{2}}\right),
$$

其中 $N$ 是展开式项数, $a$ 为光阑孔径, $A_{j}$ 和 $B_{j}$ 是复 常数, 分别表示为展开系数和高斯系数 ${ }^{[16]}$. 通过计 算可得 LG 光束在有光阑限制的光学系统中的传输 公式:

$$
\begin{aligned}
& E(r, \theta, z) \\
& =\sum_{j=1}^{N} A_{j}\left\{\frac{k}{B} \exp (-\mathrm{i} k z) \mathrm{i}^{l+1} \exp (-\mathrm{i} l \theta) \times \exp \left(-\frac{\mathrm{i} k p r^{2}}{2 B}\right)\right. \\
& \times \int_{0}^{\infty} \exp \left(-\frac{B_{j} r_{0}^{2}}{a^{2}}\right) \times\left(\frac{\sqrt{2} r_{0}}{w_{0}}\right)^{l} L_{p}^{l}\left(\frac{2 r_{0}^{2}}{w_{0}{ }^{2}}\right) J_{l}\left(\frac{k r r_{0}}{B}\right)
\end{aligned}
$$




$$
\left.\times \exp \left(-\frac{\mathrm{i} k A r_{0}^{2}}{2 B}-\frac{r_{0}^{2}}{w_{0}^{2}}\right) r_{0} \mathrm{~d} r_{0}\right\},
$$

其中 $J_{l}\left(\frac{k r r_{0}}{B}\right)$ 是 $l$ 阶贝塞尔函数. $A, B$ 和 $D$ 是由光阑 与透镜构成的 $A B C D$ 光学系统的矩阵元, 当入射空 间和出射空间的折射率相同时, 满足 $A D-B C=1$.

\section{2 光轨道角动量密度及量子特性}

光束的轨道角动量源于光束横截面内的线动量 的角向分量 ${ }^{[17]}$, 沿光束传播方向的轨道角动量密度 可由径矢 $r$ 叉乘光束横截面内的线动量密度的 $\phi$ 分量 得到, 即

$$
M_{z}=\left|\left(r \times \varepsilon_{0}\langle E \times B\rangle\right)_{z}\right|=\varepsilon_{0}\left|r \times\langle E \times B\rangle_{\phi}\right|,
$$

其中 $r$ 为位置矢量, $\varepsilon_{0}\langle E \times B\rangle$ 为坡印廷矢量时间平 均值的实部, 即光束横截面内的线动量密度, \langle\rangle 表 示时间的平均, $\varepsilon_{0}$ 为真空介电常数, $E$ 为电场强度 矢量, $B$ 为磁感应强度矢量. 对一沿 $\hat{x}$ 方向偏振的矢 势可以得到 ${ }^{[18]}$ :

$$
\varepsilon_{0}\langle E \times B\rangle=\mathrm{i} \omega \frac{\varepsilon_{0}}{2}\left(u \nabla u^{*}-u^{*} \nabla u\right)+\omega k \varepsilon_{0}|u|^{2} \hat{z},
$$

其中 $\omega$ 为角频率, $u$ 是近轴传播模式下光场的复振 幅, $u^{*}$ 是 $u$ 的复共轭, $k$ 为波数. 将(7)式代入(6)式, 可得光束的轨道角动量密度为

$$
M_{z}=\mathrm{i} \omega \frac{\varepsilon_{0}}{2}\left\{x\left[u \frac{\partial u^{*}}{\partial y}-u^{*} \frac{\partial u}{\partial y}\right]-y\left[u \frac{\partial u^{*}}{\partial x}-u^{*} \frac{\partial u}{\partial x}\right]\right\} .
$$

在柱坐标系下将(5)式作为 $u$ 的数学表达式代入 (8)式, 得到 $L G$ 光束通过含光阑系统后接收面上的光 轨道角动量密度分布函数为

$$
M_{z}=\mathrm{i} \omega \frac{\varepsilon_{0}}{2}\left(u \frac{\partial u^{*}}{\partial \theta}-u^{*} \frac{\partial u}{\partial \theta}\right) .
$$

利用态函数反映单光子系统的状态, 系统在该 状态下的典型物理性质可通过相应的态矢量函数来 表示, 量子数代表本征方程中态函数所描写的状态 的信息, 通过求解厄米算符的本征方程解得本征值, 即所谓的量子数, 就能够把相应的态函数所载的信 息提取出来. 轨道角动量密度对应于波函数的空间 分布, 引入轨道角动量密度算符 $\hat{L}_{z}$ 作用于分布函数 $M_{z}$, 则 $\mathrm{LG}$ 光束的光子轨道角动量密度算符
$\hat{L}_{z}=-\mathrm{i} \hbar \frac{\partial}{\partial \theta}$ 的本征值方程为 ${ }^{[19]}$

$$
\hat{L}_{z}\left|M_{z}{ }^{L G}\right\rangle=(l \hbar)\left|M_{z}{ }^{L G}\right\rangle,
$$

其中 $\left|M_{z}{ }^{L G}\right\rangle$ 为算符 $\hat{L}_{z}$ 的本征态, $i \hbar$ 为算符 $\hat{L}_{z}$ 的本征 值, 不同的本征值对应不同的本征态. 得到单光子轨 道角动量密度在某一范围空间分布的概率为

$$
\left.P\left(l_{n}\right)=|| M_{z}^{L G}\right\rangle\left._{n}\right|^{2} .
$$

\section{3 仿真分析}

对 1 阶空心 $L G$ 模式浴旋光束通过含单光阑及薄 透镜的光学系统进行仿真计算, 在一定接收距离处 根据接收面场强分布以及光束轨道角动量密度变化 情况, 分析光阑对浴旋光束传输特性的影响. 计算中 取 LG 光束径向量子数 $p=0$, 即径向波节数为 1 . 弧 向量子数 $l=1$. LG 光束的传输方向为 $z$ 轴正方向, 在 $z=0$ 处放置一个半径为 $a$ 的圆孔光阑, 光束束腰 $w_{0}$ 位于光阑处, 在光阑平面后一定距离处放置一薄透 镜, 透镜焦距 $F=0.6 \mathrm{~m}$, 接收面距光源距离 $2 \mathrm{~m}$. 光学 系统的物理模型如图 1 所示.

图 1(a)为透镜紧靠光阑光学系统, 图 1(b)为透镜 与光阑分离光学系统, 透镜与光阑间距 $s=0.2 \mathrm{~m}$. 分 别基于两种物理模型对波长 $\lambda=632.8 \mathrm{~nm}$ 及 $\lambda=1.06 \mu \mathrm{m}$ 两种 $\mathrm{LG}$ 浴旋光束进行仿真分析, 束腰半径 $w_{0}=5 \mathrm{~mm}$, 以光阑孔径 $a$ 与束腰半径 $w_{0}$ 之比 $a / w_{0}$ 作为遮拦比, 分析遮拦比等参数的变化对光束传输的影响.

\section{1 透镜紧靠光阑光学系统}

(i ) 图 2 为取光束波长 $\lambda=632.8 \mathrm{~nm}$, 不同遮拦比 $a / w_{0}$ 下接收面的光强分布情况.

从图 2 可以看出, 当遮拦比 $a / w_{0}<1.8$ 时, 光阑系 统遮拦比对 LG 通过光阑系统的场强分布影响明显, 接收面接收到的光斑由暗到亮逐渐趋于完整, 当遮 拦比 $a / w_{0} \geqslant 1.8$ 以后，场强分布开始表现稳定. 同时发 现系统遮拦比主要对光斑强度变化有影响, 对光束 束宽变化影响不大.

(ii) 图 3 为取波长 $\lambda=1.06 \mu \mathrm{m}$, 不同遮拦比 $a / w_{0}$ 下接收面的光强分布情况.

从图 3 可以看出, 当遮拦比 $a / w_{0}<2.6$ 时, 系统遮 拦比对 LG 通过光阑系统的场强分布影响随着遮拦 

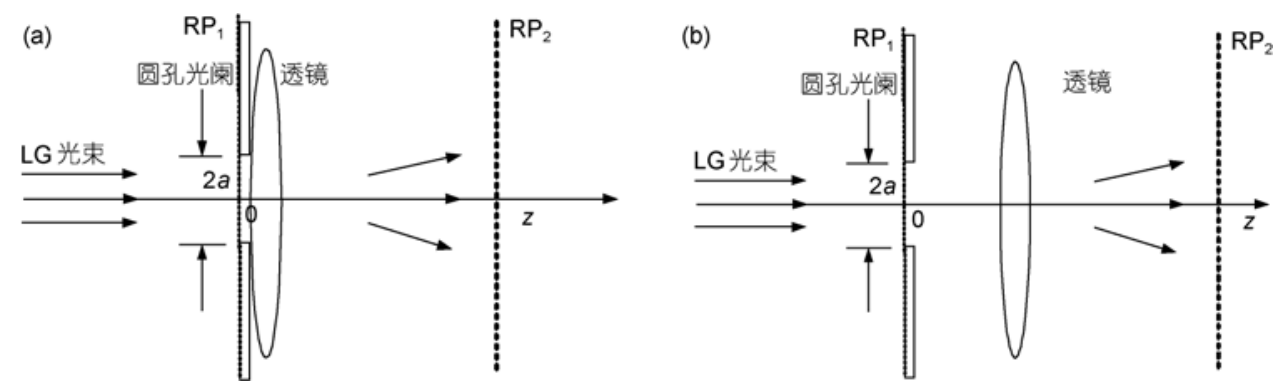

图 1 LG 光束通过单光阑光学系统的物理模型

(a) 透镜紧靠光阑; (b) 透镜光阑分离

Figure 1 Physical model of the LG beam through single aperture optical system. (a) Lens close to the aperture; (b) lens and the aperture separating.
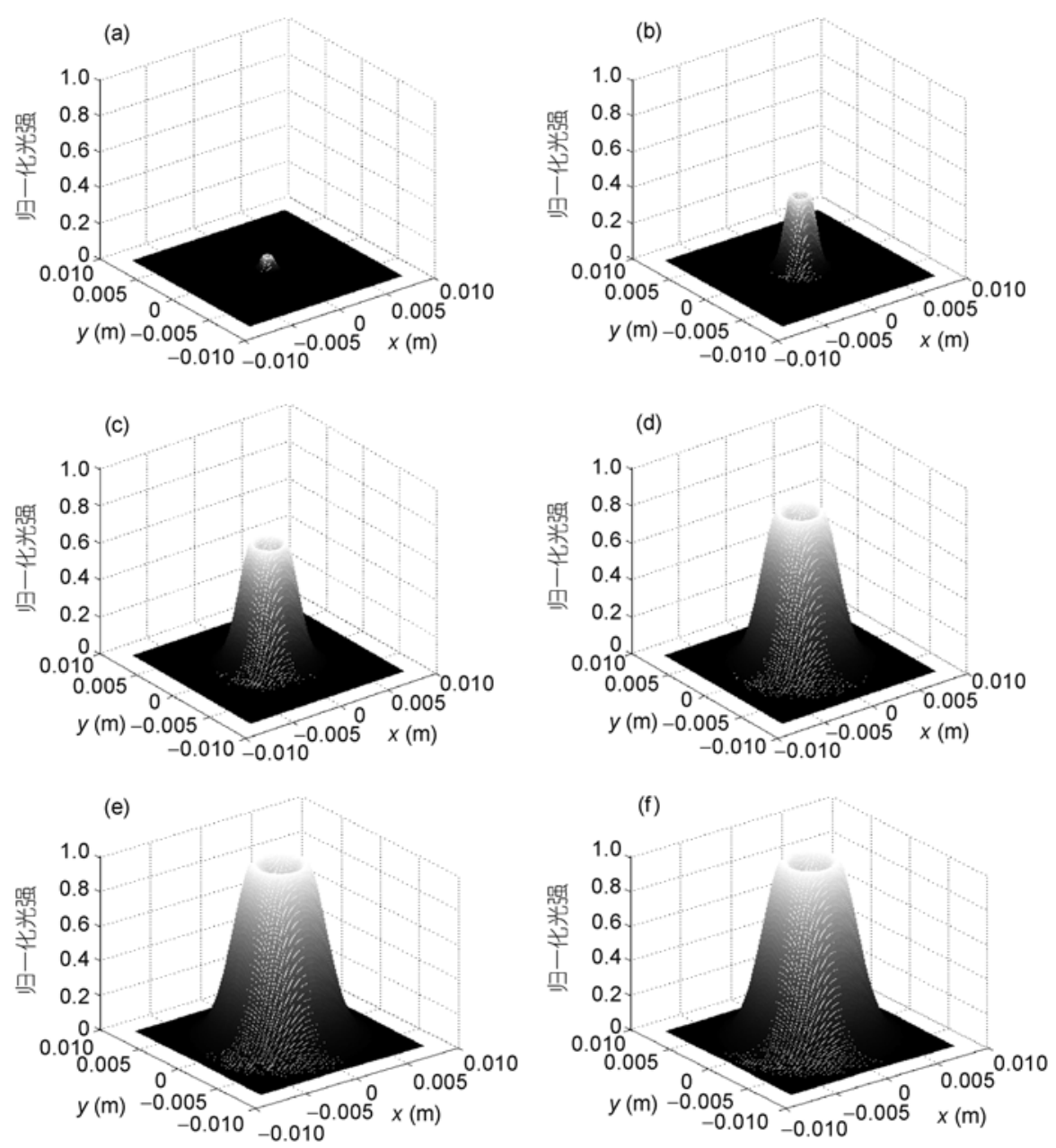

图 2 不同遮拦比 $a / w_{0}$ 下 $\lambda=632.8 \mathrm{~nm} L G_{0}^{\mathbf{1}}$ 光束光强分布

(a) $a / w_{0}=0.2 ;$ (b) $a / w_{0}=0.8 ;$ (c) $a / w_{0}=1.2 ;$ (d) $a / w_{0}=1.6$; (e) $a / w_{0}=1.8$; (f) $a / w_{0}=2.0$

Figure 2 The intensity distribution of $L G_{0}^{1}$ beam $(\lambda=632.8 \mathrm{~nm})$ at difirrent obscuration ratio. (a) $a / w_{0}=0.2$; (b) $a / w_{0}=0.8 ;$ (c) $a / w_{0}=1.2$; (d) $a / w_{0}=1.6$; (e) $a / w_{0}=1.8$; (f) $a / w_{0}=2.0$. 

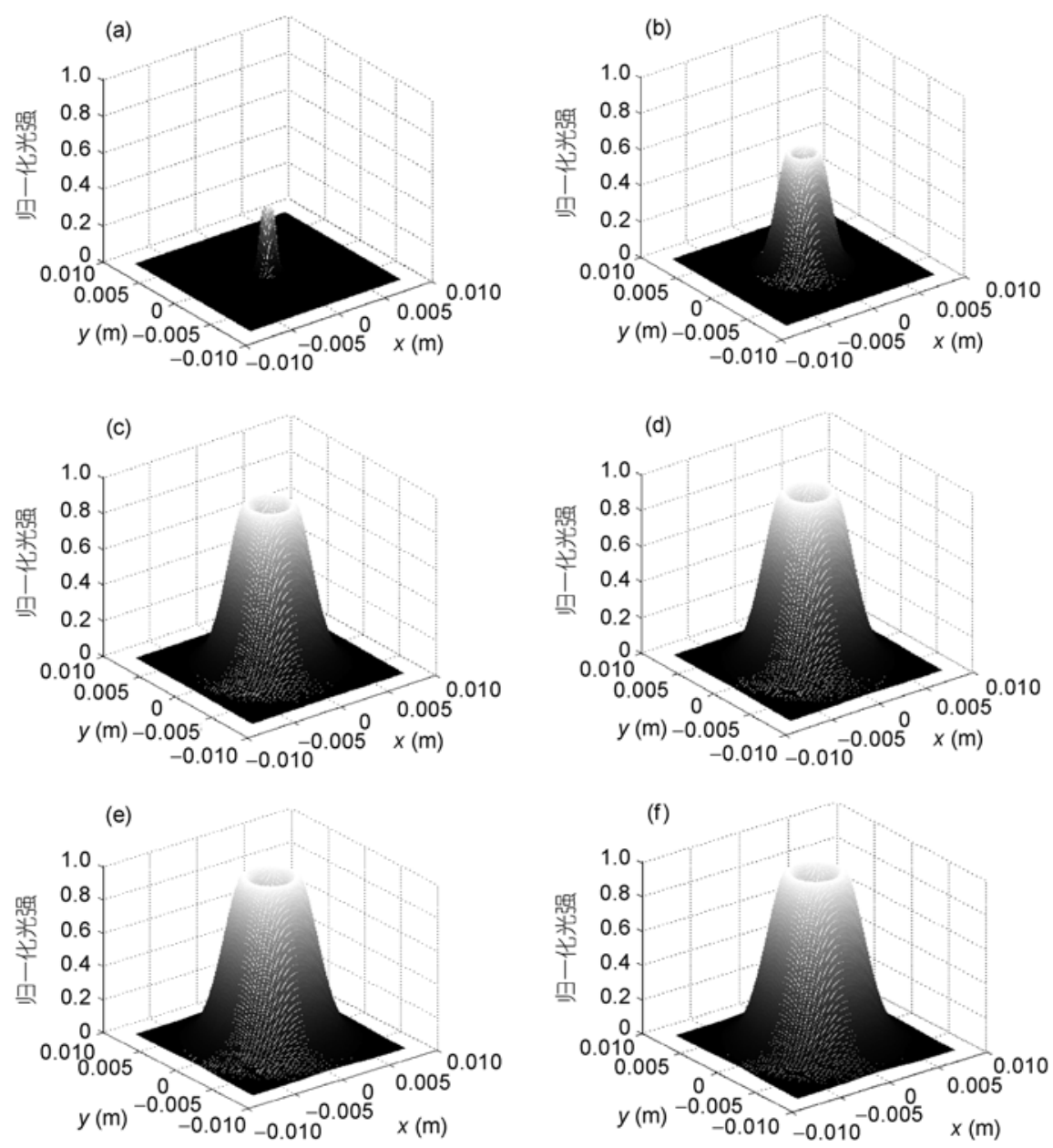

图 3 不同遮拦比 $a / w_{0}$ 下 $\lambda=1.06 \mu \mathrm{m} L G_{0}^{1}$ 光束光强分布

(a) $a / w_{0}=0.2 ;$ (b) $a / w_{0}=0.8 ;$ (c) $a / w_{0}=1.6$; (d) $a / w_{0}=1.8 ;$ (e) $a / w_{0}=2.6$; (f) $a / w_{0}=3.5$

Figure 3 The intensity distribution of $L G_{0}^{1}$ beam $(\lambda=1.06 \mu \mathrm{m})$ at difirrent obscuration ratio. (a) $a / w_{0}=0.2$; (b) $a / w_{0}=0.8 ;$ (c) $a / w_{0}=1.6$; (d) $a / w_{0}=1.8$; (e) $a / w_{0}=2.6$; (f) $a / w_{0}=3.5$.

比增加逐渐减小, 接收面接收到的光斑由暗逐渐变 亮, 当遮拦比 $a / w_{0} \geqslant 2.6$ 以后, 场强分布开始表现稳定, 而接收光束束宽不受遮拦比变化的影响. 对比两种 不同波长 $\mathrm{LG}$ 光束通过光阑的传输特性, 发现波长 $\lambda=1.06 \mu \mathrm{m}$ 时遮拦比对光束传输影响比波长 $\lambda=632.8$ $\mathrm{nm}$ 时要大, 前者 $a / w_{0} \geqslant 2.6$ 以后接收面光场分布开始 保持稳定不变, 后者 $a / w_{0} \geqslant 1.8$ 时光阑效应可忽略.

\section{2 透镜-光阑分离光学系统}

(i ) 计算中取波长 $\lambda=632.8 \mathrm{~nm}$, 透镜与光阑 间距 $s=0.2 \mathrm{~m}$, 图 4 为不同遮拦比 $a / w_{0}$ 下的光强分布 情况.

从图 4 可以看出, 当遮拦比 $a / w_{0}<2.2$ 时光阑系统
遮拦比对 LG 光束的场强分布影响明显, 接收面接 收到的光斑逐渐由暗到亮趋于完整, 当遮拦比 $a / w_{0} \geqslant 2.2$ 以后, 场强分布开始表现稳定. 系统遮拦比 主要对光斑强度变化有影响, 对光束束径变化影响 不大.

(ii) 计算中取波长 $\lambda=1.06 \mu \mathrm{m}$, 图 5 为不同遮拦 比 $a / w_{0}$ 下接收面的光强分布情况.

从图 5 可以看出, 遮拦比 $a / w_{0}<3.4$ 时光阑系统遮 拦比对 LG 通过光阑系统的场强分布影响明显, 接收 面接收到的光斑由暗到亮, 遮拦比 $a / w_{0} \geqslant 3.4$ 以后, 场 强分布表现稳定, 光阑系统对光束传输不再产生影 响. 同时发现系统遮拦比主要对光斑强度变化有影 响, 对光束束宽变化影响很小. 

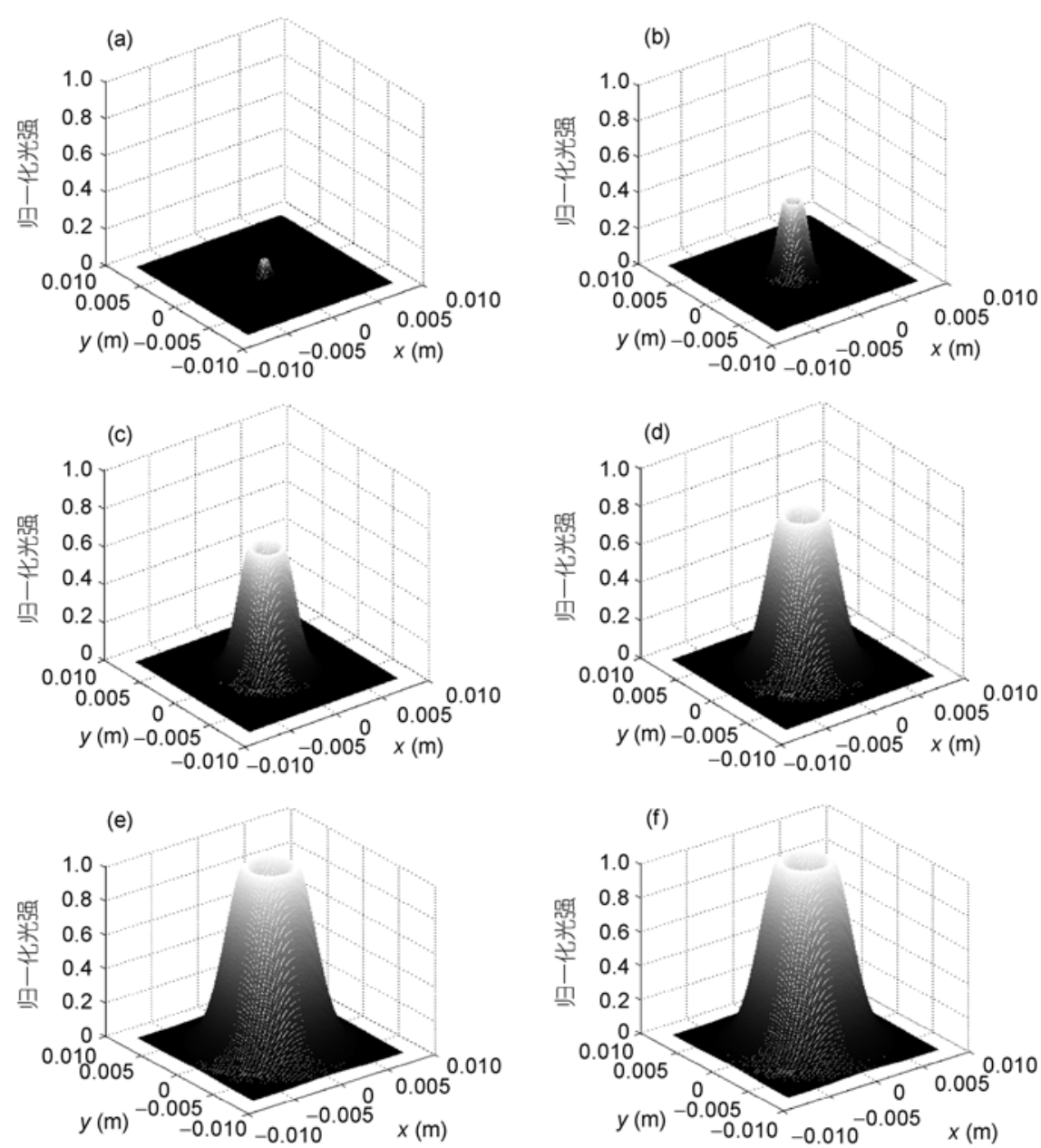

图 4 不同遮拦比 $a / w_{0}$ 下 $\lambda=632.8 \mathrm{~nm} L G_{0}^{1}$ 光束光强分布

(a) $a / w_{0}=0.2$; (b) $a / w_{0}=0.8$; (c) $a / w_{0}=1.6$; (d) $a / w_{0}=1.8$; (e) $a / w_{0}=2.2$; (f) $a / w_{0}=3.5$

Figure 4 The intensity distribution of $L G_{0}^{1}$ beam $(\lambda=632.8 \mathrm{~nm})$ at difirrent obscuration ratio. (a) $a / w_{0}=0.2$; (b) $a / w_{0}=0.8$; (c) $a / w_{0}=1.6$; (d) $a / w_{0}=1.8 ;$ (e) $a / w_{0}=2.2$; (f) $a / w_{0}=3.5$.

分别对波长为 $\lambda=1.06 \mu \mathrm{m}$ 与 $\lambda=632.8 \mathrm{~nm} \mathrm{LG}$ 光束 通过光阑的场分布分析发现, 对光阑与薄透镜分离 的光学系统, $L G$ 光束在波长 $\lambda=1.06 \mu \mathrm{m}$ 时的遮拦比对 光束传输影响比 $\lambda=632.8 \mathrm{~nm}$ 时大, 当遮拦比 $a / w_{0} \geqslant 3.4$ 以后光阑系统对光束传输影响不明显, 而 $\lambda=632.8$ $\mathrm{nm}$ 时遮拦比 $a / w_{0} \geqslant 2.2$ 光阑孔径效应消失. 将两种光 学系统物理模型对比分析发现, 在近场接收范围内, 随着透镜与光阑分离距离加大, 光阑孔径遮拦比对 光束传输影响会增大, 例如光束波长 $\lambda=632.8 \mathrm{~nm}$ 时, 光阑紧靠透镜情况下遮拦比 $a / w_{0} \geqslant 1.8$ 后对光束不会 产生影响, 而光阑与透镜分离情况下遮拦比 $a / w_{0} \geqslant 2.2$ 后光阑孔径效应消失. 在相同物理模型中, 比较 $\lambda=632.8 \mathrm{~nm}$ 与 $\lambda=1.06 \mu \mathrm{m}$ 两种波长光束发现, 波长较 大的光束在同一遮拦比下衍射效应比较明显, 随着 遮拦比增大, 衍射效应减弱, 当遮拦比 $a / w_{0} \geqslant 1.8$ 时光 阑透镜系统的衍射效应可以忽略，此时接收面横向 场强分布基本保持不变，可保证携带信息的 LG 浴旋 光束的有效传输.

在上述束腰半径 $w_{0}=5 \mathrm{~mm}$ 的基础上, 取不同束 腰光束进行仿真计算, 结果发现, 在同一物理模型下, 光阑孔径遮拦比对相同波长不同束腰半径浴旋光束 传输影响程度有所区别, 当遮拦比 $a / w_{0}<0.8$ 时, 束腰 半径较小的光束接收面衍射效应比束腰半径大的光 束明显, 当遮拦比 $a / w_{0}>0.8$ 以后, 不同束腰半径光束 

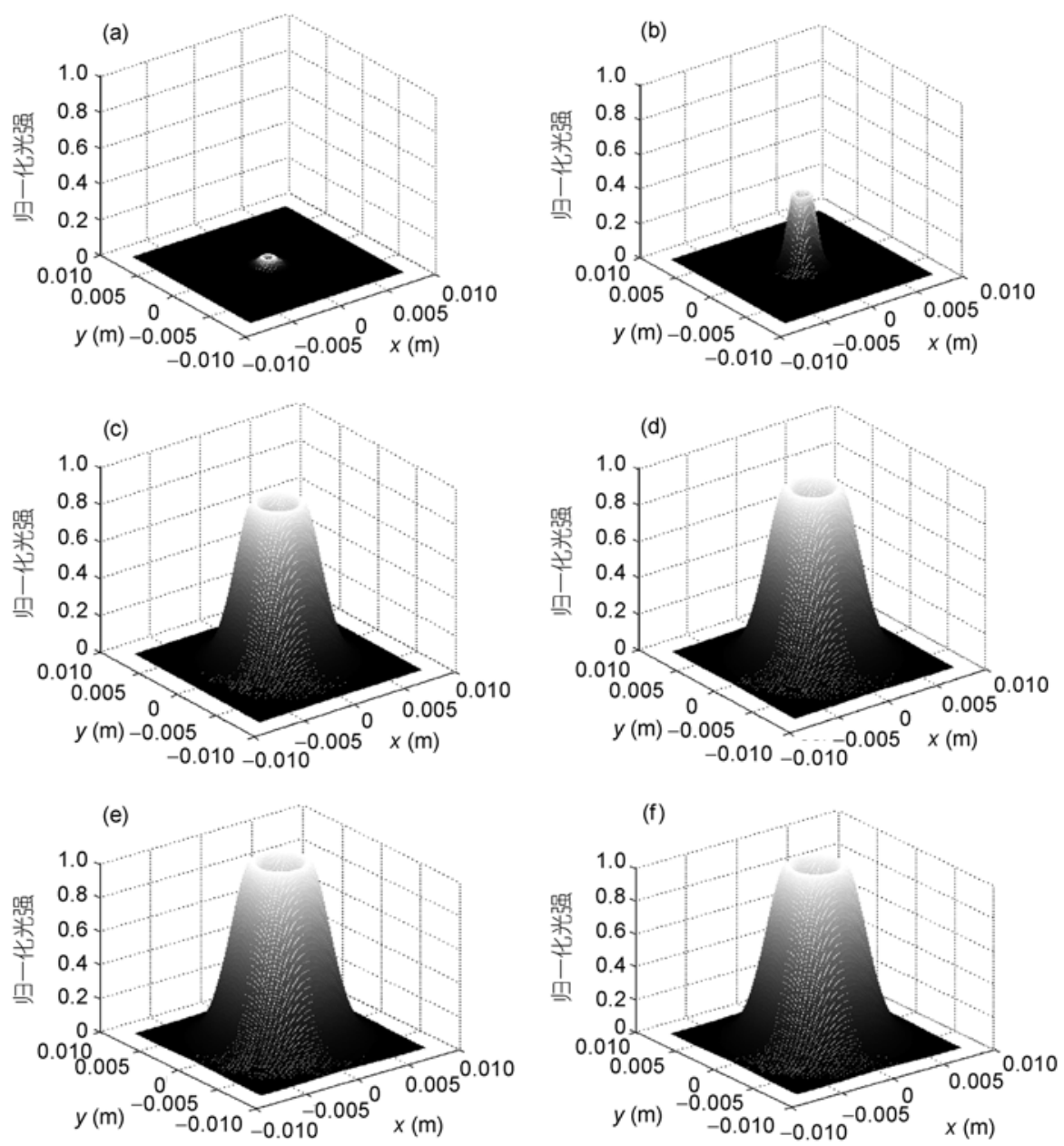

图 5 不同遮拦比 $a / w_{0}$ 下 $\lambda=1.06 \mu \mathrm{m} L G_{0}^{1}$ 光束光强分布

(a) $a / w_{0}=0.2$; (b) $a / w_{0}=0.8$; (c) $a / w_{0}=1.5$; (d) $a / w_{0}=2$; (e) $a / w_{0}=3.4$; (f) $a / w_{0}=6$

Figure 5 The intensity distribution of $L G_{0}^{1}$ beam $(\lambda=1.06 \mu \mathrm{m})$ at difirrent obscuration ratio. (a) $a / w_{0}=0.2 ;$ (b) $a / w_{0}=0.8 ;$ (c) $a / w_{0}=1.5$; (d) $a / w_{0}=2$; (e) $a / w_{0}=3.4$; (f) $a / w_{0}=6$.

在接收面衍射效应趋于一致, 总体上不同的束腰半 径对接收面场强分布变化影响很小.

图 6 为光阑紧靠透镜的情况下, 波长 $\lambda=632.8 \mathrm{~nm}$ 时不同遮拦比 $a / w_{0}$ 下 $L G_{0}^{1}$ 光束近轴接收面光强随径 向距离 $r$ 变化的场分布, 束腰半径 $w_{0}=5 \mathrm{~mm}$. 从图 6 中可以看出, 接收平面上光场关于中心原点呈圆对 称环状分布, 光阑孔径对暗中空 $L G_{0}^{1}$ 光束的光场分 布有很大的影响, 当光阑孔径远小于束腰半径, 取遮 拦比为 $a / w_{0}=0.2$ 时, 光斑中心光强为零, 由于仍有部 分光子通过光阑系统, 在接收面上会有相对较暗光 斑, 光斑最大光强值很小. 随着遮拦比的增加, 当 $a / w_{0}>1.5$ 时, 光斑轴上光强近似为零, 光束空心性明
显, 此时光场分布趋于稳定, 当遮拦比 $a / w_{0} \geqslant 1.8$ 光场 分布稳定, 光束传输基本不受光阑及透镜系统的影 响. 从图 6 中还可以看出, 光环的内外半径基本不受 光阑孔径影响.

对光諫紧靠透镜的物理模型, 计算中通过单独 调节光束波长、接收横截面距离等参数, 观察结果基 本相同, 说明了由于相位奇点引起的空心性使得 LG 光束的强度分布在一定传播的过程中不发生变化, 即在一定距离的传播过程中, 暗中心可一直保持, 光 束在近场空间传播时有很好的传输稳定性. 对于光 束远场的传输, 则需要分别在水平传输和斜程传输 情况下, 考虑诸如湍流因素等大气环境的影响. 


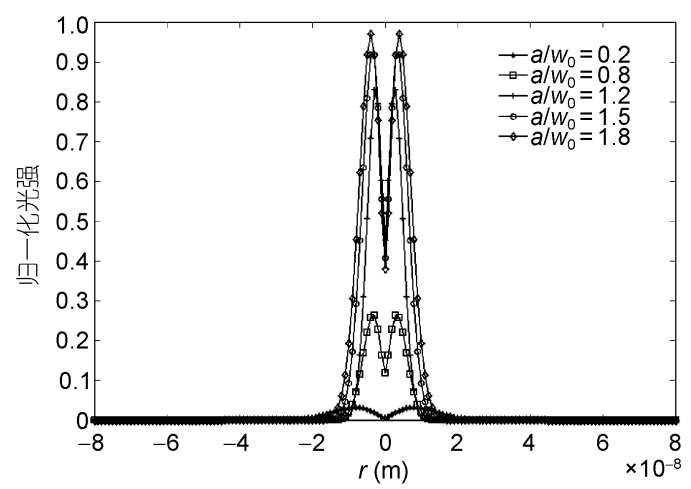

图 6 不同遮拦比 $a / w_{0}$ 下 $w_{0}=5 \mathrm{~mm} L G_{0}^{1}$ 光束近轴 $2 \mathrm{~m}$ 处 光强分布

Figure 6 The intensity distribution of paraxial $L G_{0}{ }^{1}$ beam $\left(w_{0}=5 \mathrm{~mm}\right)$ at $2 \mathrm{~m}$ distance at difirrent obscuration ratio.

图 7 为光阑紧靠透镜的情况下, 波长 $\lambda=1.06 \mu \mathrm{m}$ 时不同遮拦比下接收横截面光斑相位分布, 由于 $L G_{0}^{1}$ 暗中空光束相位分布函数中含有一个与旋转方 位角 $\theta$ 成正比的相位因子 $\exp (\mathrm{i} l \theta)$, 图 7 中显示了在 同一接收面某确定方向上浴旋场相位随方位角 $\theta$ 变 化曲线. 从图中可以看出, 对于 1 阶 $\mathrm{LG}$ 浴旋光束, 在 通过含单光阑薄透镜光学系统后光束的相位分布基 本没有受到系统遮拦比的影响, 光束在近场空间传 播时轨道角动量不会发生变化.
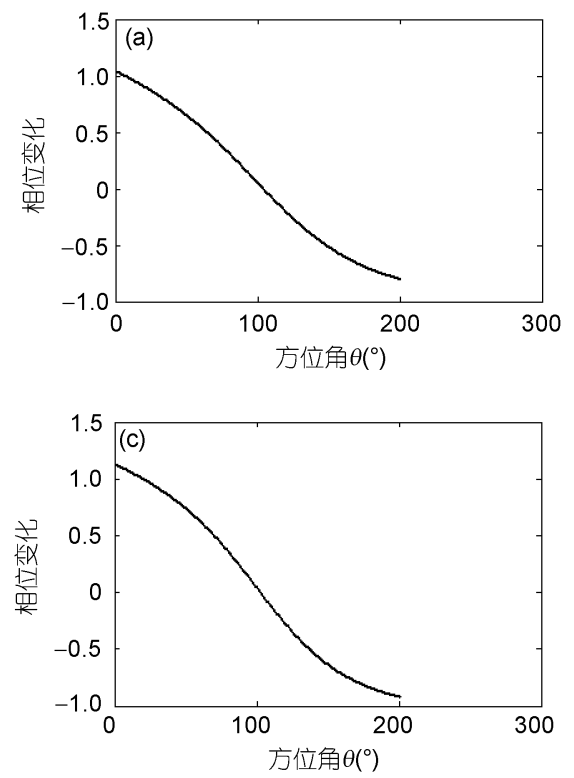

对光阑紧靠透镜的情况下取 $L G_{0}^{1}$ 光束波长 $\lambda=632.8 \mathrm{~nm}, 1$ 阶 $\mathrm{LG}$ 光束轨道角动量密度受遮拦比 $a / w_{0}$ 变化影响曲线如图 8 所示, 从图 8 中可以看出, 在垂直于光束传播方向的同一接收面上, 轨道角动 量密度随着遮拦比的增加呈递增趋势, 当遮拦比 $a / w_{0}>1.8$ 时趋于稳定, 不会随着遮拦比的增加而增大, 此时光阑系统对光束轨道角动量密度的影响为零.

计算中还对光束传输距离 $z \leqslant 10 \mathrm{~m}$ 的不同接收位 置处进行了仿真分析，在这个过程中同时对透镜与 光阑之间的距离进行了调节, 最终计算发现, 所得结 果与文中设计的两种典型光学系统仿真结果近似.

\section{4 结论}

研究了具有轨道角动量的 LG 浴旋光束通过受圆 孔硬边光阑限制的 $\mathrm{ABCD}$ 光学系统的传输特性, 推 导了柱坐标下 $L G$ 光束单光子轨道角动量密度, 结合 量子理论对力学量算符作用于单光子轨道角动量密 度函数的本征方程进行了分析. 取 LG 模式的弧向特 征量子数 $l=1$, 对 $L G_{0}^{1}$ 空心光束通过含单光阑薄透镜 光学系统进行数值仿真, 分析了孔径遮拦比对光束 近场一定传输距离场强分布及轨道角动量密度变化 的影响. 结果表明:
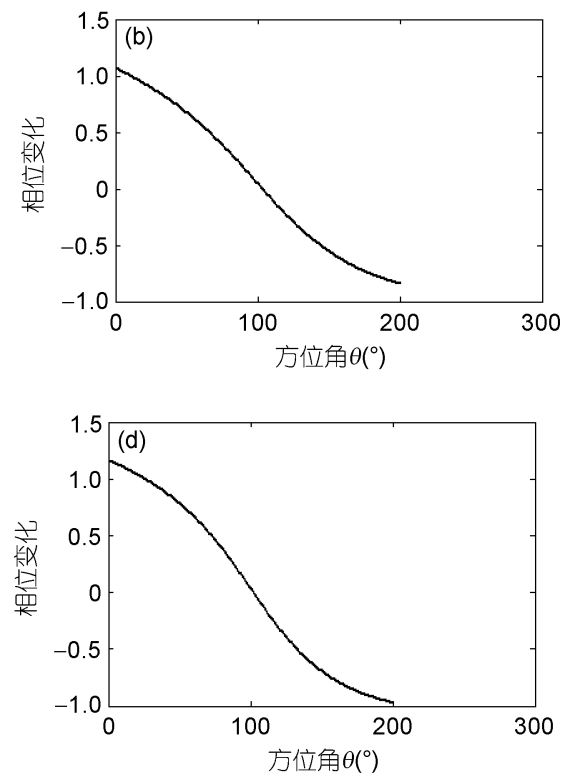

图 7 不同遮拦比 $\boldsymbol{a} / \boldsymbol{w}_{\mathbf{0}}$ 下 $\boldsymbol{L} \boldsymbol{G}_{\mathbf{0}}^{\mathbf{1}}$ 光束相位变化

(a) $a / w_{0}=0.2$; (b) $a / w_{0}=0.8$; (c) $a / w_{0}=1$; (d) $a / w_{0}=1.5$

Figure 7 The phase change of $L G_{0}^{1}$ beam at difirrent obscuration ratio. (a) $a / w_{0}=0.2 ;$ (b) $a / w_{0}=0.8$; (c) $a / w_{0}=1$; (d) $a / w_{0}=1.5$. 


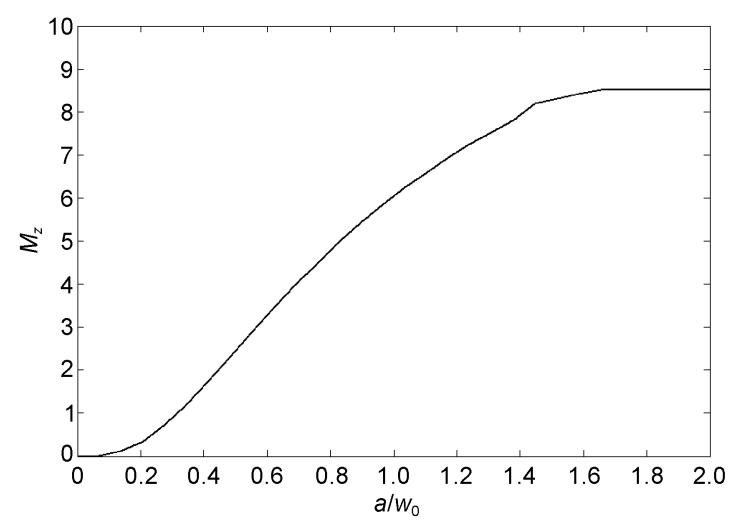

图 8 不同遮拦比 $a / w_{0}$ 下 $L G_{0}^{1}$ 光束轨道角动量密度变化 Figure 8 Changes in the orbital angular momentum density of $L G_{0}^{1}$ beam at difirrent obscuration ratio.

(i) 确定透镜光阑系统物理模型后, 光阑孔径 效应的影响程度与光束的波长有关, 波长增大系统 遮拦比对光束传输的影响程度变大; 在相同波长 下, 光束束腰半径的变化对光阑孔径效应的影响贡 献不大. (ii) 将透镜-光阑分离光学系统与透镜紧靠光阑 光学系统两种物理模型进行对比分析发现，波长相 同时随着透镜与光阑之间的距离增加, 透镜-光阑分 离光学系统遮拦比对光束传输影响随之增大.

(iii) 在同一透镜光阑系统物理模型中, 随着光 阑系统遮拦比的增加光束轨道角动量密度也增大, 到一定程度趋于稳定. 例如光阑紧靠透镜系统中, 当 光束波长 $\lambda=632.8 \mathrm{~nm}$ 、遮拦比 $a / w_{0}>1.8$ 时, 轨道角动 量密度趋于稳定.

(iv) 在浴旋光束通过透镜光阑系统近场传输中, 系统遮拦比对浴旋光束相位分布的影响不明显, 光 束束径在传输过程中基本保持不变, 光束轨道角动 量变化不大.

通过调整不同接收距离进行仿真分析，结果与 文中设计的两种典型光学系统仿真结果基本近似, 表明 $L G_{0}^{1}$ 暗中空浴旋光束在近场空间传播时有很好 的传输稳定性, 本文研究结果可为浴旋光束轨道角 动量用于光通信提供一定的理论基础.

\section{参考文献}

1 Molina-Terriza G, Torres J P, Torner L. Management of the angular momentum of light: Preparation of photons in multidimensional vector states of angular momentum. Phys Rev Lett, 2002, 88: 013601

2 Torner L, Torres J P, Carrasco S. Digital spiral imaging. Opt Express, 2005, 13(3): 873-881

3 Neil A T, MacVicar I, Allen L, et al. Intrinsic and extrinsic nature of the orbital angular momentum of a light beam. Phys Rev Lett, 2002, 88(5): 053601

4 Cullet P, Gil L, Rocca F. Optical vortices. Opt Commun, 1989, 73: 403-408

5 Allen L, Lembessis V E, Babiker M. Spin-orbit coupling in free-space Laguerre-Gaussian light beam. Phys Rev A, 1996, 53(5): R2937R2939

6 Gibson G, Courtial J, Padgett M J. Free-space information transferusing light beams carrying orbital angular momentum. Opt Express, 2004, 12(22): 5448-5456

7 Inoue R, Kanai N, Yonehara T, et al. Entanglement of orbital angular momentum states between an ensemble of cold atoms and a photon. Phys Rev A, 2006, 74: 053809

8 Lv H, Ke X Z. Research on the beam with orbital angular momentum used in encoding and decoding of optical communication (in Chinese). Acta Opt Sin, 2009, 29(2): 331-335 [吕宏, 柯熙政. 具轨道角动量光束用于光通信编码及解码研究. 光学学报, 2009, 29(2): 331-335]

9 Wang X Q, Lv B D. Focusing properties of Laguerre-Gaussian beams (in Chinese). Laser Tech, 1996, 20(3): 185-190 [王喜庆, 吕百达. 拉 盖尔-高斯光束的聚焦特性. 激光技术, 1996, 20(3): 185-190]

10 Peng R W, Lv B D. Focused field characteristics and focal shift of Laguerre-Gaussian beams passing through an aperture-lens separation system (in Chinese). High Power Laser Particle Beams, 2002, 14(4): 541-544 [彭润伍, 吕百达. 拉盖尔-高斯光束通过光阑-透镜分离系 统的聚焦特性和焦移. 强激光与粒子束, 2002, 14(4): 541-544]

11 Ge W G, Ma S J, Liu Y X, et al. Transformation of Laguerre-Gaussian beams through an optical system with recurrence aperture (in Chinese). Laser J, 2006, 27(2): 36-37 [葛卫国, 马善钧, 刘永欣, 等. 拉盖尔-高斯光束通过含矩形光阑光学系统的变换. 激光杂志, 2006, 27(2): 36-37]

12 Wang B Z, Zhao Z G, Duan K L. Propagation properties of hollow Gaussian beams through an optical system with a hard-edged aperture (in 
Chinese). Laser Tech, 2007, 31(2): 203-205, 208 [王备战, 赵志国, 段开椋. 空心高斯光束通过光阑透镜系统的传输特性. 激光技术, 2007, 31(2): 203-205, 208]

13 Gao C Q, We G H, Horst W. Orbital angular momentum of the laser beam and the second order intensity moments. Sci China Ser A-Math Phys Astron, 2000, 43(12): 1306-1311

14 Collins S A. Lens-system diffraction integral written in terms of matrix optics. Opt Soc Am A, 1970, 60(7): 1168-1177

15 Qing Y S, Lv B D. Propagation of Laguerre-Gaussian beams through an optical system with hard edge aperture (in Chinese). Laser Tech, 2002, 26(3): 174-176 [卿与三, 吕百达. 拉盖尔-高斯光束通过有硬边光阑光学系统的传输. 激光技术, 2002, 26(3): 174-176]

16 Wen J J, Breazeale M A. A diffraction beam field expressed as the super-position of Gaussian beams. Acoust Soc Am, 1988, 83(5): $1752-1756$

17 Gao M W, Gao C Q, Lin Z F. Generation of twisted stigmatic beam and transfer of orbital angular momentum during the beam transformation (in Chinese). Acta Phys Sin, 2007, 56(4): 2184-2190 [高明伟, 高春清, 林志锋. 扭转对称光束的产生及其变换过程中的 轨道角动量传递. 物理学报, 2007, 56(4): 2184-2190]

18 Allen L, Beijersbergen M W, Spreeuw R J C, et al. Orbital angular momentum of light and the transformation of the Laguerre-Gaussian laser modes. Phys Rev A, 1992, 45: 8185-8189

19 Ke X Z, Lu N, Yang Q L. Research of transmission characteristics of single-photon orbital angular momentum. Acta Phys Sin, 2010, 59(9): 6159-6063 [柯熙政, 卢宁, 杨秦岭. 单光子轨道角动量的传输特性研究. 物理学报, 2010, 59(9): 6159-6063]

\title{
Effects of aperture on the orbital angular momentum of hollow vortex beam
}

\author{
LÜ Hong \& KE XiZheng* \\ Faculty of Automation and Information Engineering; Xi'an University of Technology, Xi'an 710048, China
}

Based on the expended method of complex Gaussion functions, the recurrence propagation formulae of LaguerreGaussian (LG) beams with orbital angular momentum (OAM) through optical system with aperture is studied. The photon orbital angular momentum density function and state vector of LG beam are derived in cylindrical coordinates. The light intensity distribution of the first order LG beam through optical system with aperture was numerically simulated. The effects of the obscuration ratio on the propagation characteristics of different wavelength LG beams based on two optical physical models with an aperture, and the phase distribution and changes in the orbital angular momentum density of LG beam propagating through optical system were studied. Results show that the effects of the obscuration ratio on the propagation characteristics of LG beams are closely related to the wavelength. The obscuration ratio has much effect on the orbital angular momentum density, and the waist radius of LG beams has no obvious effect on the orbital angular momentum. These results provide some theoretical bases for the vortex beam with orbital angular momentum used in encoding of free space optical communication.

beam propagation, optical vortices, orbital angular momentum, aperture

PACS: 42.60.Jf, 42.50.Tx, 42.79.Ag

doi: $10.1360 / 132010-1149$ 